\title{
Overcoming the transcatheter aortic valve replacement Achilles heel: coronary re-access
}

\author{
Arash Arshi, Steven J. Yakubov, Kevin L. Stiver, Carlos E. Sanchez \\ OhioHealth Riverside Methodist Hospital, Columbus, Ohio, USA \\ Correspondence to: Arash Arshi, MD. OhioHealth Physician Group, 3705 Olentangy River Road, Suite 100, Columbus, OH 43214, USA. \\ Email: arash.arshi@ohiohealth.com.
}

\begin{abstract}
Transcatheter aortic valve replacement (TAVR) is an alternative to surgical aortic valve replacement (SAVR) for the treatment of symptomatic severe aortic stenosis (AS). Coronary artery disease (CAD) is common in patients with severe AS. As the indications for TAVR extend to lower risk patients with longer life expectancy and as CAD is a progressive condition, coronary angiography will become increasingly common in patients who have had a previous TAVR. Coronary artery re-access after TAVR may be challenging but is possible in most cases. Commissural alignment of the prosthesis with the native coronary ostia plays an important role in successful coronary re-access. Coronary artery obstruction is a potentially devastating complication of TAVR, particularly in valve-in-valve procedures. In the present keynote lecture, we review techniques used to mitigate the risk of coronary obstruction, as well as catheter selection and strategies for selective coronary artery engagement for specific transcatheter aortic valve (TAV) bioprostheses.
\end{abstract}

Keywords: Aortic stenosis (AS); coronary artery disease (CAD); transcatheter aortic valve replacement (TAVR)

Submitted Mar 05, 2020. Accepted for publication Jul 09, 2020.

doi: $10.21037 /$ acs-2020-av-38

View this article at: http://dx.doi.org/10.21037/acs-2020-av-38

\begin{abstract}
Introduction
Transcatheter aortic valve replacement (TAVR) has revolutionized the management of patients with severe aortic stenosis (AS). Initial clinical trials established TAVR as the standard of care for patients at prohibitive surgical risk (1), and as an alternative to surgical aortic valve replacement (SAVR) in patients at high and intermediate risk for surgery (2-5). Subsequent trials demonstrated the safety and efficacy of TAVR in low risk patients, with results comparable (6) or superior to surgery (7). In 2017, for the first time in the United States, the number of patients with severe AS treated with TAVR surpassed the number treated with surgery.
\end{abstract}

\section{Coronary artery disease and aortic stenosis}

Concomitant coronary artery disease (CAD) in severe AS is common. The prevalence of obstructive CAD in patients undergoing TAVR is approximately $50 \%$, although the rate is steadily declining as indications for TAVR now include healthier patients (8). Two meta-analyses have suggested that percutaneous coronary intervention (PCI) to treat severe CAD prior to TAVR does not confer any clinical advantage $(9,10)$. However, another study has shown that more complete revascularization, as determined by residual SYNTAX score, may improve outcomes in patients with severe CAD who undergo TAVR (11). Despite the conflicting data, the authors of the 2017 Appropriate Use Criteria guidelines for PCI suggest that PCI is nearly always appropriate or may be appropriate in patients with severe CAD undergoing TAVR, even with low risk findings on noninvasive testing (12). Justifications for PCI prior to TAVR include the comprehensive treatment of cardiac disease, reducing ischemic risk during valve intervention, especially if rapid pacing is needed and simplifying coronary intervention without interference from a valve prosthesis.

Both patients and physicians tend to choose the less invasive treatment option when outcomes are similar, and TAVR is now generally preferred to surgery in the 
absence of prohibitive anatomic features across the entire risk spectrum. Furthermore, as risks and recovery time associated with TAVR have decreased, the threshold to recommend TAVR may continue to lower over time. Studies are underway to evaluate TAVR in patients with asymptomatic severe AS in the EARLY TAVR trial (Evaluation of Transcatheter Aortic Valve Replacement Compared to Surveillance for Patients With Asymptomatic Severe Aortic Stenosis; NCT03042104) and in patients with moderate AS and symptomatic heart failure in the TAVR UNLOAD trial (Transcatheter Aortic Valve Replacement to Unload the Left Ventricle in Patients With Advanced Heart Failure; NCT02661451). As the average age and severity of comorbidities of patients undergoing TAVR decreases, patients will be living longer and more active lives following their valve intervention. This creates the potential need for subsequent coronary angiography and intervention in TAVR patients. Patients may develop new onset or progressive CAD, and restenosis of previously placed stents may require invasive evaluation and treatment. The rate of post-TAVR acute coronary syndrome has been reported to be as high as $10 \%$ over two years (13).

Coronary angiography post TAVR will likely be increasingly common. Due to the anatomic relation of the coronary ostia to the prosthetic aortic valve, coronary artery re-access may be affected. Unique design characteristics of the type of transcatheter aortic valve (TAV) must also be considered when developing a strategy to engage the coronary arteries. Numerous trials demonstrate the feasibility of coronary angiography and intervention postTAVR (14-23), but with consistently lower reported success rates following implantation of the Medtronic CoreValve (Medtronic Inc., Minneapolis, MN, USA), especially of the right coronary artery (RCA) $(14,17,19,21,22)$. In the current paper, we review valve deployment strategies to optimize re-access to the coronary arteries, focusing on valve-in-valve procedures, as well as technique and catheter selection to aid in successful post-TAVR angiography and intervention.

\section{Commissural alignment of TAVs}

Preprocedural planning with multidetector computed tomography (CT) angiography is essential in assessing aortic valve anatomy and the potential risk of coronary obstruction peri- and post-TAVR. Likewise, consideration of patient specific anatomical factors for future coronary reaccess in low- and intermediate-risk patients has become a topic of debate for potential TAV-in-TAV indication.
Anatomical factors impacting coronary re-access postTAVR include coronary ostia height, sinus of Valsalva width and sinotubular junction width and height.

More recently, device specific factors, such as device positioning and orientation during deployment, have been described as aspects impacting coronary re-access post-TAVR. In particular, commissural tab orientation or commissural alignment of TAV in relation to the coronary ostia takeoff, has recently garnered attention. During SAVR, the calcified leaflets are removed and the bioprosthetic surgical aortic valve (SAV) is sutured to the annulus, aligning the commissural frame posts to the native commissures to avoid commissural post overlap with the coronary ostia. During TAVR the commissural posts cannot be reliably aligned to the native aortic valve commissures and posts can land directly in front of the coronary ostia, potentially impeding coronary re-access.

Tang et al. first described the impact of the geometrical orientation of TAVs, including the CoreValve Evolut selfexpanding valve (Medtronic PLC, Minneapolis, MN, USA) and Sapien 3 balloon-expandable valve (Edwards Lifesciences, Irvine, CA, USA), during initial deployment in relation to their neo-commissural alignment and the risk of overlap with coronary artery ostia (24). The CoreValve Evolut R and Pro valves have three commissural frame posts that measure $26 \mathrm{~mm}$ in height, with one of the posts aligned with the $\mathrm{C}$-tab paddle on the valve frame. Aligning the commissural posts away from the coronary artery ostia during TAVR is unpredictable. The study by Tang et al. found that the hat orientation on the delivery catheter can help define the valve position during deployment and may predict the commissural alignment orientation in relation to the coronary ostia. When the hat marker of the CoreValve Evolut delivery system was oriented at the outer curve and center front of the aorta on the coplanar three-cusp angiographic view (Figure 1A), severe coronary overlap of the left anterior descending artery, RCA or both with the commissural posts carried the lowest incidence at $23.2 \%$. However, when the hat marker was oriented towards the inner curve and center back of the aorta on the same coplanar view (Figure 1B), the frequency of coronary overlap with a commissural post was higher at $75 \%$ (24). Controlling TAV commissural alignment can prove to be important, especially in lower-risk and younger patients with severe AS presenting with concomitant symptomatic CAD or acute coronary syndromes, where unrestricted access to coronary arteries during repeat coronary angiography is relevant. Therefore, it is recommended to 

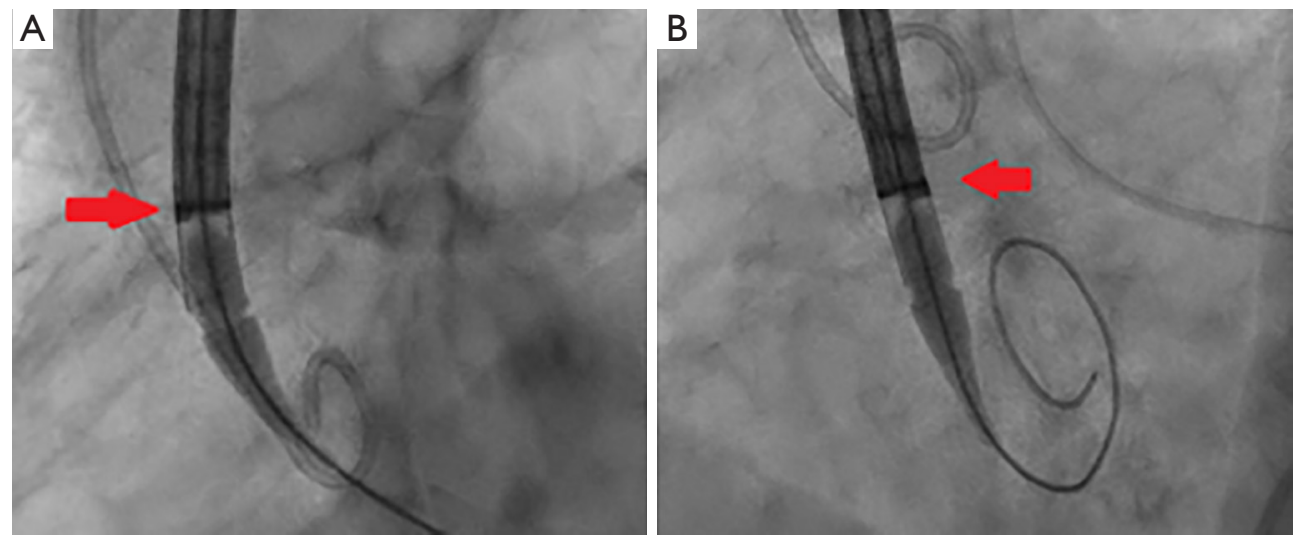

Figure 1 Hat marker orientation of the Medtronic CoreValve Evolut Delivery system in relationship to the curvature of the aorta. Hat orientation (red arrow) on the outer curvature of the aorta in the left anterior oblique (A). Hat orientation (red arrow) on the inner curvature of the aorta in the left anterior oblique view (B).

introduce the CoreValve Evolut R/Pro delivery system into the common femoral artery in a standardized manner with flush ports directed to the three o'clock position. This orientation ensures that the hat marker faces the outer curve of the aortic root as the delivery system traverses the aortic valve on the three-cusp coplanar angiographic view, helping to achieve proper commissural alignment and avoid overlap with the coronary ostia.

The design of the Sapien 3 differs from that of the CoreValve Evolut in that instead of a commissural post, there is a $3 \mathrm{~mm}$ pledget or tab located on the commissural upper row cells (Figure 2) (25). Although the frame height of the Sapien 3 valve is shorter than the Evolut valve, the commissural tabs may end up in front of the coronary ostia, especially if the coronary takeoff is low (Figure 3). For example, coronary ostia with a height of $10 \mathrm{~mm}$ or less will be covered by a Sapien 3 commissural tab if the valve implant is positioned $90 \%$ aortic or higher (25). Intentional commissural alignment of the Sapien 3 valve is currently not possible by crimping the valve at different specific orientations.

\section{Prevention of coronary artery obstruction}

Coronary obstruction during TAVR in a SAV is more common than that during TAVR in a native valve, occurring in $3-4 \%$ of procedures (26). Commissural posts of surgical valves are typically aligned with those of the native valve. There is usually no compromise of coronary access unless the sinuses are unusually effaced and the ostia of the coronary arteries are exceptionally low.
Risk factors for coronary obstruction include female sex, narrow sinus of Valsalva width $(<30 \mathrm{~mm})$ and ostial height $<10 \mathrm{~mm}$ (27). CT angiography is essential for measuring aortic root dimensions, especially of the bioprosthetic valve to coronary artery (VTC) distance. Final VTC distance of $<3 \mathrm{~mm}$ is associated with a high risk of coronary obstruction (26). Strategies to prevent obstruction include laceration of leaflets and/or guide catheter protection of the endangered coronary ostia during the valve deployment procedure. Bioprosthetic or native aortic scallop intentional leaflet laceration to prevent iatrogenic coronary obstruction (BASILICA) prior to TAVR (28) has become a preventative measure in high-risk cases. This catheter based guidewire technique involves electrocautery of a stiff guidewire to pierce the base of the aortic valve cusp. The guidewire is then snared in the left ventricle, creating a wire loop. A non-insulated, uncoated part of the wire is placed at the leaflet and electrocautery is applied again, splitting the aortic leaflet. Upon deployment of the transcatheter valve, the bioprosthetic or native leaflet is splayed to try to limit coronary obstruction. Excessive leaflet calcification or thickening may be more challenging for adequate leaflet laceration. Similar challenges may occur due to unique bioprosthetic valve design differences.

Commissural alignment for commercially available TAV systems is not only important for future coronary reaccess, but also for future TAVR-in-TAV planning. Special attention to aortic root anatomy, dimensions, coronary height and TAV frame orientation on CT angiography is absolutely necessary to assess the risk of coronary occlusion when a TAVR-in-TAV is considered. In patients 

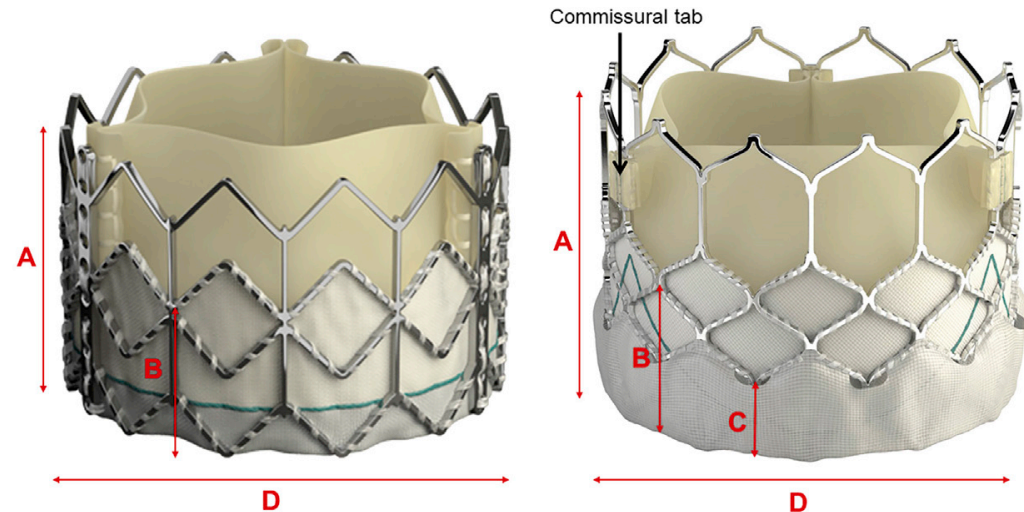

\begin{tabular}{|c|c|c|c|c|c|c|c|}
\hline & $\begin{array}{c}23 \mathrm{~mm} \\
\text { Sapien } \\
\text { XT }\end{array}$ & $\begin{array}{c}26 \mathrm{~mm} \\
\text { Sapien } \\
\text { XT }\end{array}$ & $\begin{array}{c}29 \mathrm{~mm} \\
\text { Sapien } \\
\text { XT }\end{array}$ & $\begin{array}{c}20 \mathrm{~mm} \\
\text { Sapien } 3\end{array}$ & $\begin{array}{l}23 \mathrm{~mm} \\
\text { Sapien } 3\end{array}$ & $\begin{array}{c}26 \mathrm{~mm} \\
\text { Sapien } 3\end{array}$ & $\begin{array}{l}29 \mathrm{~mm} \\
\text { Sapien } 3\end{array}$ \\
\hline A. Frame Height & $14 \mathrm{~mm}$ & $17 \mathrm{~mm}$ & $19 \mathrm{~mm}$ & $15.5 \mathrm{~mm}$ & $18 \mathrm{~mm}$ & $20 \mathrm{~mm}$ & $22.5 \mathrm{~mm}$ \\
\hline $\begin{array}{l}\text { B. Inner Skirt } \\
\text { Height }\end{array}$ & $6.7 \mathrm{~mm}$ & $8.7 \mathrm{~mm}$ & $11.6 \mathrm{~mm}$ & $7.9 \mathrm{~mm}$ & $9.3 \mathrm{~mm}$ & $10.2 \mathrm{~mm}$ & $11.6 \mathrm{~mm}$ \\
\hline $\begin{array}{l}\text { C. Outer Skirt } \\
\text { Height }\end{array}$ & $\mathrm{N} / \mathrm{A}$ & N/A & N/A & $5.2 \mathrm{~mm}$ & $6.6 \mathrm{~mm}$ & $7.0 \mathrm{~mm}$ & $8.1 \mathrm{~mm}$ \\
\hline $\begin{array}{l}\text { D. Valve } \\
\text { Diameter }\end{array}$ & $23 \mathrm{~mm}$ & $26 \mathrm{~mm}$ & $29 \mathrm{~mm}$ & $20 \mathrm{~mm}$ & $23 \mathrm{~mm}$ & $26 \mathrm{~mm}$ & $29 \mathrm{~mm}$ \\
\hline
\end{tabular}

Figure 2 Balloon-expandable valves: features and dimensions. Various dimensions of the Sapien XT and Sapien 3 Valves (Edwards Lifesciences, Irvine, California) are listed for comparison. Reprinted from ref (25), with permission from Elsevier.
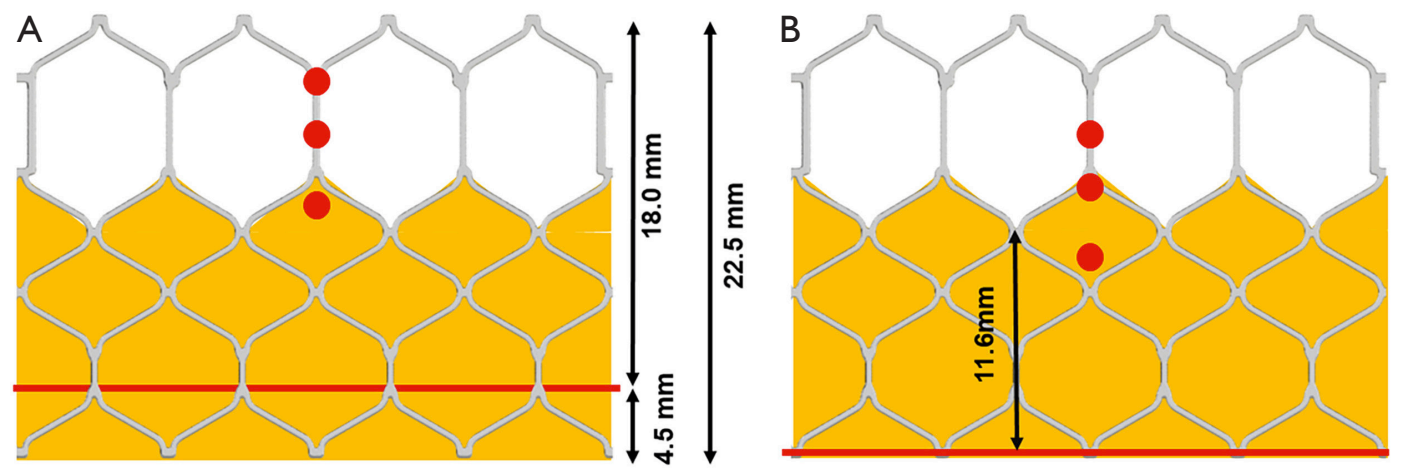

Figure 3 Balloon-expandable and coronary ostia based on depth of implant. Red dot represents the different locations of the coronary ostium in relation to the valve frame of a $29 \mathrm{~mm}$ Sapien 3 Valve (Edwards Lifesciences, Irvine, California), and the red line represents the annular plane. An optimally positioned Sapien 3 valve (Edwards Lifesciences, Irvine, California) (A) would make the coronary access potentially easier than one with a higher implant (B), where the coronary ostium will be located below the seal skirt. Tall native leaflet or bulky calcium at the leaflet tip may further increase difficulty of coronary access in a high valve implant. Reprinted from ref (25), with permission from Elsevier.

at high risk for coronary occlusion with TAVR-in-TAV, BASILICA can help prevent coronary artery obstruction and facilitate coronary re-access if necessary. However, if the TAV commissural tab is overlapping the coronary ostia, intentional laceration of the transcatheter heart valve (THV) leaflet will not be effective in the prevention of coronary artery obstruction.

Other techniques have been described for preventing 
coronary obstruction in those at high risk of requiring TAVR in SAV. The "chimney snorkel" is one technique whereby the coronary artery is engaged with a guide catheter and guide extender prior to TAVR in SAV (29). The catheter valve system is deployed, followed by stenting the coronary artery and extending the stent above the bioprosthetic valve. The proximal part of the stent is flared to improve flow and to allow for easier coronary access if the artery needs to be engaged again in the future. Additional techniques to minimize coronary obstruction include selecting valves with larger stent cell sizes to allow easier coronary re-access, using TAV types with lower sealing skirt or direct anchoring of the TAV to the bioprosthetic aortic valve to minimize leaflet mobility and obstruction (30).

Palmerini et al., evaluated patients at high risk of coronary obstruction during TAVR who underwent wiring of coronary arteries pre-TAVR or were protected with preventive coronary stenting (31). Preventive stenting was associated with favorable survival at three years compared to protective wiring alone. There was a slight excess of myocardial infarction in those with protective stenting, but a higher occurrence of delayed coronary occlusion in those with protective wiring.

\section{Coronary angiography and intervention after TAVR}

Angiographic imaging of the coronary arteries begins with the understanding of the location of the coronary arteries with respect to the TAV. The geometric configuration of the TAV often dictates initial catheter selection for coronary cannulation. This is assisted by an initial ascending aortogram, usually in a left anterior oblique (LAO) projection, in order to visualize the origin of the left main coronary artery (LMC) and RCA. It should be expected that valve geometry may affect the choice of catheter and technique of cannulation for each of the coronary arteries.

\section{Coronary access after TAVR in bicuspid aortic valves}

Coronary angiography in patients with bicuspid valves undergoing TAVR can be challenging, and understanding the location of the raphe is important. The location of the raphe between the right and left coronary cusps results in asymmetric valve expansion allowing ample space between the valve frame and coronary ostia. Coronary access is made easier by large native sinuses, which is common in bicuspid valve patients. A wider sinotubular junction also aids coronary access by enhancing coronary flow around the valve frame and improving ostial access (32). Often, a high implantation of the TAV is desirable for bicuspid valves; however, this approach may pose a technical challenge with sealing skirt interference for coronary re-access, especially in patients with low coronary artery height.

\section{Coronary access with medtronic CoreValve Evolut}

The self-expanding CoreValve Evolut R/Pro has a diamondshaped cell nitinol frame design with three components (Figure 4). The inflow provides hoop strength and radial force and is covered by tissue or a sealing skirt to minimize paravalvular leak. There is a constrained central part and an outflow part of the frame that extends above the sinotubular junction. A key feature of coronary access is to introduce the diagnostic or guiding catheter at the constrained part of the valve (Figure 5). This is above the sealing skirt, which is $13 \mathrm{~mm}$ from the bottom of the frame. An initial aortogram can give insight into the best cell to choose for engagement and show the coronary height in relation to the valve. Sometimes, the aortogram may be all that is necessary to verify adequate flow in the major vessels.

An algorithm for diagnostic catheter selection and guide catheter selection has been proposed by Yudi et al. (25). Challenges in access arise in two particular instances. One is when the valve implantation is high and the coronary artery height is normal or low. In this instance, engagement of the coronary artery will occur from a diamond cell that is higher than the coaxial plane of the coronary artery. If the catheter is not able to selectively engage the ostium, a coronary wire may be passed into the artery to act as a rail to engage the catheter. This may be aided by passing a balloon catheter or guide extender over the coronary wire if needed. The other challenging scenario occurs when the commissural post of the CoreValve Evolut Pro aligns with the ostium of the coronary artery. The sealing skirt is $13 \mathrm{~mm}$ in height (14 $\mathrm{mm}$ in the $34 \mathrm{~mm}$ Evolut Pro), but the commissural insertion point is $26 \mathrm{~mm}$ in height. If any of the three commissural posts overlie the coronary ostium, coaxial engagement is especially difficult for a diagnostic catheter or guide catheter (Figure 6). In this instance, using a $0.014 \mathrm{~mm}$ support coronary wire for coronary artery engagement may be adequate to pull the catheter in place. Balloon catheters and guide extensions are also often useful in these instances.

The concave waist of the CoreValve Evolut Pro measures 20-24 $\mathrm{mm}$ in diameter, depending on the valve size. As this 


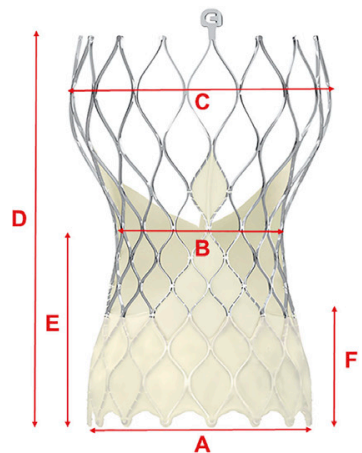

\begin{tabular}{|c|c|c|c|c|}
\hline & $\begin{array}{c}23 \mathrm{~mm} \\
\text { Evolut R I } \\
\text { PRO }\end{array}$ & $\begin{array}{c}26 \mathrm{~mm} \\
\text { Evolut R / } \\
\text { PRO }\end{array}$ & $\begin{array}{c}29 \mathrm{~mm} \\
\text { Evolut R / } \\
\text { PRO }\end{array}$ & $\begin{array}{l}34 \mathrm{~mm} \\
\text { Evolut R }\end{array}$ \\
\hline A. Inflow Diameter & $23 \mathrm{~mm}$ & $26 \mathrm{~mm}$ & $29 \mathrm{~mm}$ & $34 \mathrm{~mm}$ \\
\hline B. Waist Diameter & $20 \mathrm{~mm}$ & $22 \mathrm{~mm}$ & $23 \mathrm{~mm}$ & $24 \mathrm{~mm}$ \\
\hline C. Outflow Diameter & $34 \mathrm{~mm}$ & $32 \mathrm{~mm}$ & $34 \mathrm{~mm}$ & $38 \mathrm{~mm}$ \\
\hline D. Frame height & $45 \mathrm{~mm}$ & $45 \mathrm{~mm}$ & $45 \mathrm{~mm}$ & $46 \mathrm{~mm}$ \\
\hline E. Commissure Height & $26 \mathrm{~mm}$ & $26 \mathrm{~mm}$ & $26 \mathrm{~mm}$ & $26 \mathrm{~mm}$ \\
\hline F. Skirt Height & $13 \mathrm{~mm}$ & $13 \mathrm{~mm}$ & $13 \mathrm{~mm}$ & $14 \mathrm{~mm}$ \\
\hline
\end{tabular}

Figure 4 Repositionable self-expanding valves with and without an external pericardial wrap: features and dimensions. Various dimensions of the Evolut-R and Evolut-PRO CoreValve (Medtronic, Galway, Ireland) are listed for comparison. Reprinted from ref (25), with permission from Elsevier.
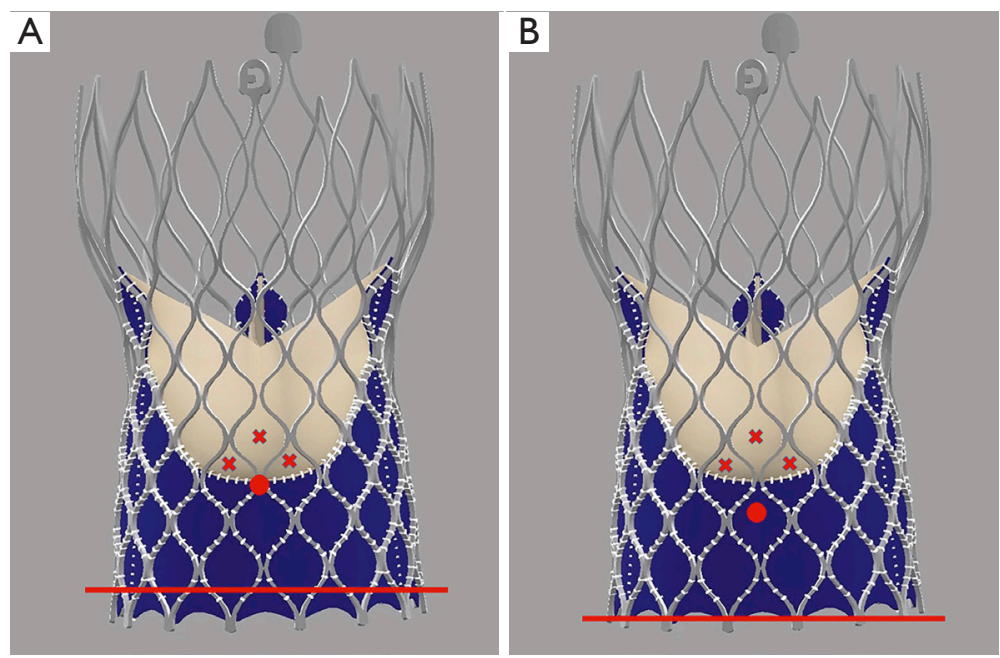

Figure 5 Self-expanding valve and coronary access depending on level of implantation across the annulus. Red dot represents the location of the coronary ostium in relation to the valve frame, and the red line represents the annular plane. The red x's depict the closest diamonds that can be used to access the coronary ostium. An optimally positioned Evolut-R (Medtronic, Galway, Ireland) (A) would make the coronary access potentially easier than the one with a higher implant (B). Reprinted from ref (25), with permission from Elsevier.

dimension is smaller than the native aortic root, alterations in catheter selection are necessary. Usually, a half-size smaller Judkins-Left (JL) catheter is more advantageous for the LMC, although longer JL catheters, where the proximal bend rests against the ascending aorta above the CoreValve waist may work as well (Figure $7 A$ ). Extra backup guide catheters can be used, but often depend on aortic root support, which is altered by the CoreValve frame and therefore, are not recommended. These guide catheters are also prone to kinking in these situations. A Judkins-Right (JR) 4 catheter is usually the best choice for the RCA, despite the smaller constrained part of the valve (Figure $7 B$ ). If the sinuses are quite large and distant from the valve frame, Amplatz-Left (AL) or Amplatz-Right (AR) catheters may be necessary for selective engagement. If the usual catheters are unable to be steered into an adequate position for coronary visualization, the Ikari guide catheters are very helpful and may be the first choice catheter for some operators. The Ikari right guide catheter can be used for the left or right coronary arteries (Figure $7 C$ ). A guide is especially useful if a coronary wire, balloon catheter or guide extender is necessary for proper engagement. 

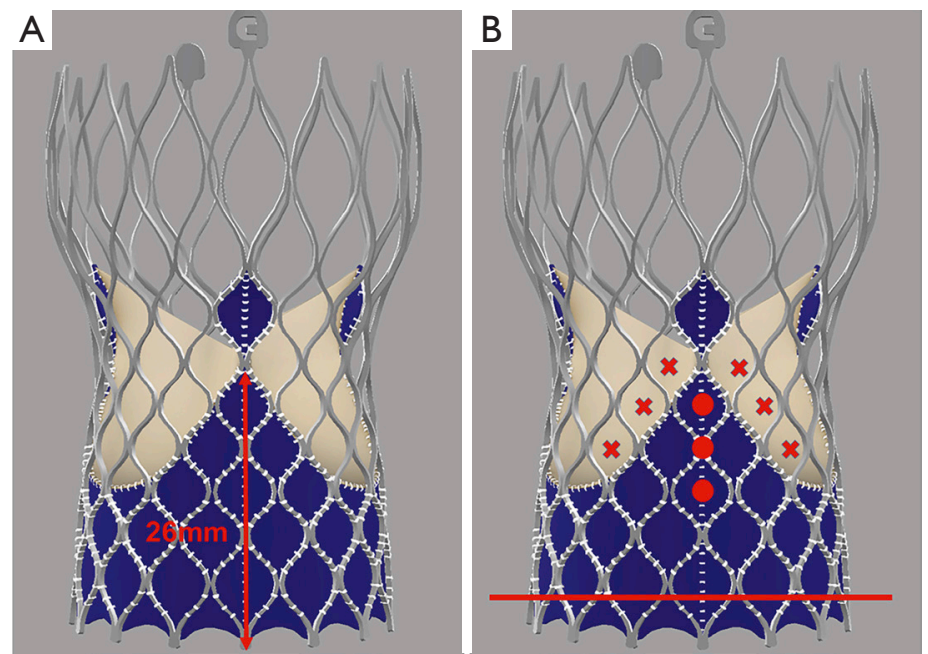

Figure 6 Self-expanding valve and coronary access if ostia lines up with commissural post. Red line represents the annular plane. The 3 red dots depict coronary ostia heights of approximately 10,14, $18 \mathrm{~mm}$ above the annular plane, respectively. The red x's depict the closest diamonds that can be used to access the coronaries. The commissural post of an Evolut-R (Medtronic, Galway, Ireland) is 26 mm in height (A). Depending on the height of the coronary ostia, a different catheter and approach is necessary for coronary reaccess, when the ostium faces the side of the commissural post (B). Reprinted from ref (25), with permission from Elsevier.
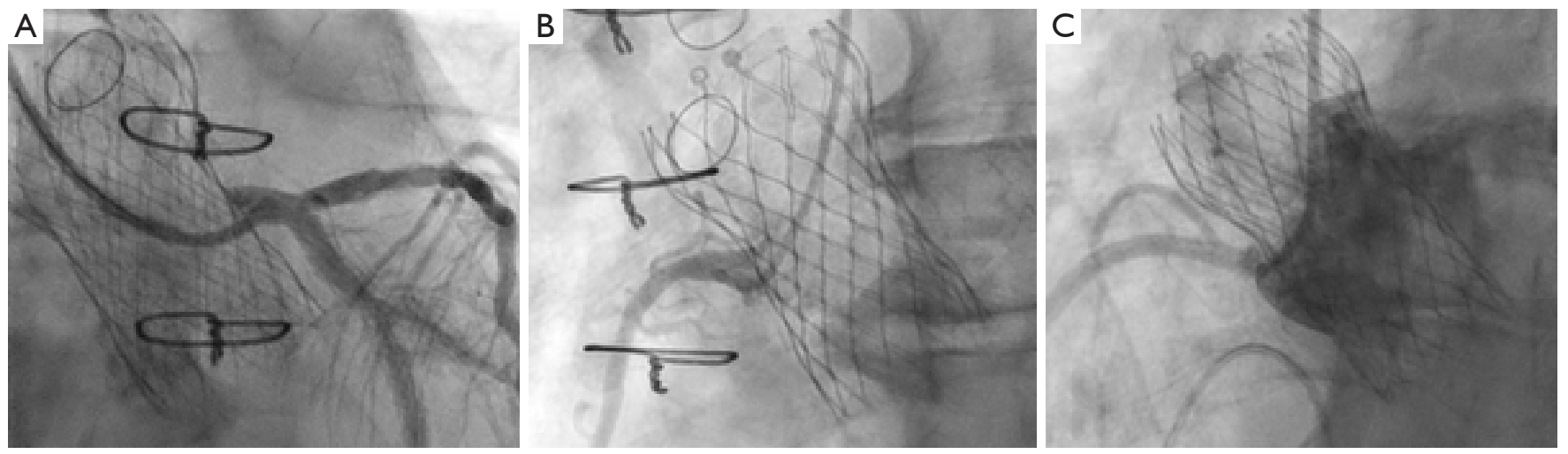

Figure 7 Coronary access within the Medtronic CoreValve Evolut R. Engagement of the left main coronary artery with a 6 French JudkinsLeft 6 guide catheter in the right anterior oblique and caudal view (A). Engagement of the right coronary artery with a 5 French JudkinsRight 5 diagnostic catheter in the left anterior oblique view (B). Engagement of the right coronary artery with a 6 French Ikari 1.5 guide catheter in the left anterior oblique view (C).

\section{Algorithm for medtronic CoreValve Evolut coronary re-access}

* Aortography with a diagnostic pigtail catheter in the outflow portion of frame in LAO projection.

* Identify the cell frame adjacent to the coronary ostia. Choose that cell or one higher to place the catheter. Always use a J-wire to introduce the catheter into a selfexpanding valve.
* From the femoral approach, standard JR4 or JL4 diagnostic catheters work well in most cases, although a half-size smaller JL catheter may be preferable.

* If catheter engagement is difficult, change catheters. Although many catheters could potentially work, the Ikari catheters seem to work exceptionally well for the right and left coronary arteries and can be considered first choice.

* If there is particular difficulty, the commissural post is 
usually aligned with the ostium of the coronary artery. Any cell above the coronary artery can be engaged and subselective angiography can be performed. If selective angiography is necessary, passing a coronary wire to the vessel and using that as a rail for introduction of the catheter is particularly helpful. This is often assisted by the use of a telescoping catheter system.

\section{Coronary artery re-access following Edwards Sapien 3}

The Edwards Sapien 3 valve has a taller frame design compared to the Sapien XT (Figure 2). Although it is more

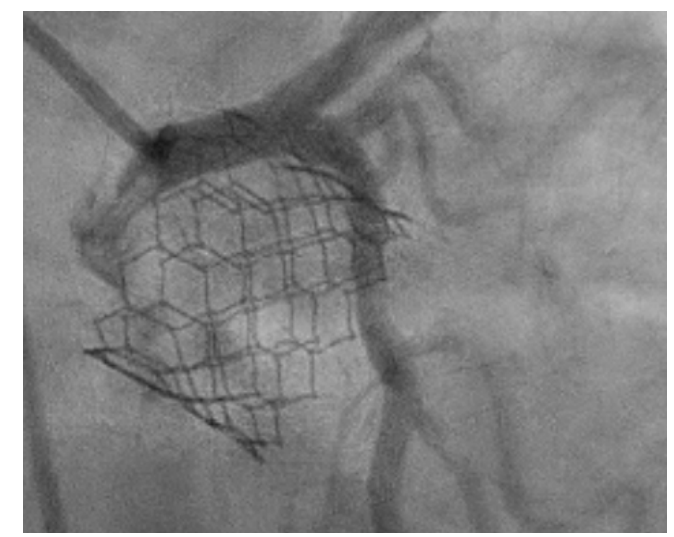

Figure 8 Coronary access within the Edwards Sapien 3 Valve. Engagement of the left main coronary artery with a 5 French Judkins-Left 3.5 diagnostic catheter in the right anterior oblique and caudal view. frequently placed above the coronary ostia, the upper cells of the frame are larger, resulting in less interference with coronary ostial access. Although it is a taller frame, it rarely extends to the level of the sinotubular junction. Even with a "high implant-90/10" deployment strategy to minimize contact with the left ventricular outflow tract in an effort to reduce pacemaker implantation rates, the risk of coronary obstruction is low. There are three commissures and 12 open cells in the frame design. Engagement of the coronary arteries through a Sapien 3 valve usually needs no alteration in catheter selection (Figure 8). Occasionally the guide catheter will need to be one size smaller. When performing PCI through the Sapien 3 valve, a guide extender is recommended in order to avoid altering the valve position or having difficulty removing the guide at the completion of the case.

\section{Coronary access following boston scientific Lotus and ACURATE neo valves}

The Lotus valve (Boston Scientific, Marlborough, MA, USA) is a mechanically expanding catheter valve system with unique coronary re-access strategies. This valve is usually deployed below the ostia of the coronary arteries. In these instances, the valve does not affect cannulation of the coronary arteries (Figure $9 A$ ) and rarely makes contact with the ascending aorta. Newer valves, such as the ACURATE neo Aortic Valve System (Boston Scientific, Marlborough, MA), have a supra-annular design, high commissural posts and a low sealing skirt, allowing easy coronary re-access due to its "free access" design architecture (Figure 9B).
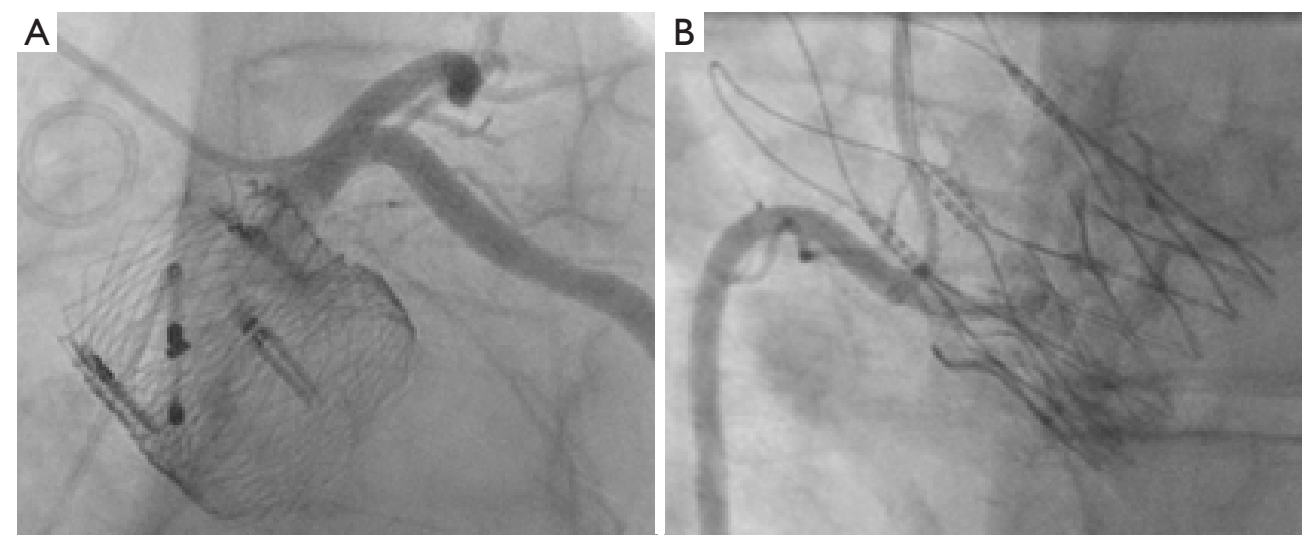

Figure 9 Coronary access within the Boston Scientific Lotus Valve and ACURATE neo Valve. Engagement of the left main coronary artery within the Lotus Valve with a 5 French Judkins-Left 5 diagnostic catheter in the left anterior oblique and caudal view (A). Engagement of the right coronary artery within a ACURATE neo Valve with a 5 French Judkins-Right 5 diagnostic catheter in the left anterior oblique view (B). 


\section{Conclusions}

Future coronary access will be an important consideration in patients undergoing TAVR. Attention to the valve design and the relation to the coronary arteries, as well as close analysis of the pre-procedure CT scan to understand the anatomy of the coronary ostia, sinus of Valsalva and sinotubular junction will help in selecting an appropriate TAV. Future TAV designs will ideally have the ability to predict and control commissural alignment with the native aortic valve. Algorithms to aid in catheter selection and optimize guide catheter engagement of the native coronary arteries post-TAVR will be helpful in the management of these patients.

\section{Acknowledgments}

Funding: None.

\section{Footnote}

Conflicts of Interest: Dr. Yakubov has received institutional grant support from Medtronic, and is on the Medical Advisory Board of Medtronic and Boston Scientific. Dr. Sanchez is a consultant for Edwards Lifesciences and Medtronic. The other authors have no conflicts of interest to declare.

Open Access Statement: This is an Open Access article distributed in accordance with the Creative Commons Attribution-NonCommercial-NoDerivs 4.0 International License (CC BY-NC-ND 4.0), which permits the noncommercial replication and distribution of the article with the strict proviso that no changes or edits are made and the original work is properly cited (including links to both the formal publication through the relevant DOI and the license). See: https://creativecommons.org/licenses/by-nc-nd/4.0/.

\section{References}

1. Leon MB, Smith CR, Mack M, et al. Transcatheter Aortic-Valve Implantation for Aortic Stenosis in Patient Who Cannot Undergo Surgery. N Engl J Med 2010;363:1597-607.

2. Smith CR, Leon MB, Mack M, et al. Transceiver versus Surgical Aortic-Valve Replacement in High-Risk Patients. N Engl J Med 2011;364:2187-98.

3. Adams DH, Popma JJ, Reardon MJ, et al. Transcatheter
Aortic-Valve Replacement with a Self-Expanding Prosthesis. N Engl J Med 2014;370:1790-8.

4. Leon MB, Smith CR, Mack M, et al. Transcatheter or Surgical Aortic-Valve Replacement in Intermediate-Risk Patients. N Engl J Med 2016;374:1609-20.

5. Reardon MJ, Van Mieghem NM, Popma JJ, et al. Surgical or Transcatheter Aortic-Valve Replacement in Intermediate-Risk Patients. N Engl J Med 2017;376:1321-31.

6. Popma JJ, Deeb GM, Yakubov SJ, et al. Transcatheter Aortic-Valve Replacement with a Self-Expanding Valve in Low-Risk Patients. N Engl J Med 2019;380:1706-15.

7. Mack MJ, Leon MB, Thourani VH, et al. Transcatheter Aortic-Valve Replacement with a Balloon-Expandable Valve in Low-Risk Patients. N Engl J Med 2019;380:1695-705.

8. Faroux L, Guimaraes L, Wintzer-Wehekind J, et al. Coronary Artery Disease and Transcatheter Aortic Valve Replacement: JACC State-of-the-Art Review. J Am Coll Cardiol 2019;74:362-72.

9. Lateef N, Khan MS, Deo SV, et al. Meta-Analysis Comparing Outcomes in Patients Undergoing Transcatheter Aortic Valve Implantation With Versus Without Percutaneous Coronary Intervention. Am J Cardiol 2019;124:1757-64.

10. Kotronias RA, Kwok CS, George S, et al. Transcatheter Aortic Valve Implantation With or Without Percutaneous Coronary Artery Revascularization Strategy: A Systematic Review and Meta Analysis. J Am Heart Assoc 2017;6:e05960.

11. Witberg, G, Regev E, Chen S, et al. The Prognostic Effects of Coronary Disease Severity and Completeness of Revascularization on Mortality in Patients Undergoing Transcatheter Aortic Valve Replacement. JACC Cardiovasc Interv 2017;10:1428-35.

12. Patel MR, Calhoon JH, Dehmer GJ, et al. ACC/AATS/ AHA/ASE/ASNC/SCAI/SCCT/STS 2017 Appropriate Use Criteria for Coronary Revascularization in Patients With Stable Ischemic Heart Disease. J Am Coll Cardiol 2017;69:2212-41.

13. Vilalta $V$, Asmarats L, Nunes Ferreira-Neto A, et al. Incidence, Clinical Characteristics, and Impact of Acute Coronary Syndrome Following Transcatheter Aortic Valve Replacement. JACC Cardiovasc Interv 2018;11:2523-33.

14. Blumenstein J, Kim WK, Liebetrau C, et al. Challenges of Coronary Angiography and Intervention in Patients Previously Treated by TAVI. Clin Res Cardiol 2015;104:632-9. 
15. Allali A, El-Mawardy M, Schwarz B, et al. Incidence, Feasibility and Outcome of Percutaneous Coronary Intervention After Transcatheter Aortic Valve Implantation with a Self-Expanding Prosthesis. Results From a Single Center Experience. Cardiovasc Revasc Med 2016;17:391-8.

16. Chakravarty T, Sharma R, Abramowitz Y, et al. Outcomes in Patients with Transcatheter Aortic Valve Replacement and Left Main Stenting: the TAVR-LM Registry. J Am Coll Cardiol 2016;67:951-60.

17. Chetcuti S, Kleiman NS, Matthews R, et al. Percutaneous Coronary Intervention After Self-Expanding Transcatheter Aortic Valve Replacement. J Am Coll Cardiol 2016;68:B300-1.

18. Zivelonghi C, Pesarini G, Scarsini R, et al. Coronary Catheterization and Percutaneous Interventions After Transcatheter Aortic Valve Implantation. Am J Cardiol 2017;120:625-31.

19. Boukantar M, Gallet R, Mouillet G, et al. Coronary Procedures After TAVI With the Self-Expanding Aortic Bioprosthesis Medtronic CoreValveTM, Not an Easy Matter. J Interv Cardiol 2017;30:56-62.

20. Htun WW, Grines C, Schreiber T. Feasibility of Coronary Angiography and Percutaneous Coronary Intervention After Transcatheter Aortic Valve Replacement Using a MedtronicTM Self-Expandable Bioprosthetic Valve. Catheter Cardiovasc Interv 2018;91:1339-44.

21. Tanaka A, Jabbour RJ, Testa L, et al. Incidence, Technical Safety, and Feasibility of Coronary Angiography and Intervention Following Self-expanding Transcatheter Aortic Valve Replacement. Cardiovasc Revasc Med 2019;20:371-5.

22. Nai Fovino L, Scotti A, Massussi M, et al. Incidence and Feasibility of Coronary Access After Transcatheter Aortic Valve Replacement. Catheter Cardiovasc Interv 2020;96:E535-41.

23. Ferreira-Neto AN, Puri R, Asmarats L, et al. Clinical and Technical Characteristics of Coronary Angiography and Percutaneous Coronary Interventions Performed Before and After Transcatheter Aortic Valve Replacement with a Balloon-Expandable Valve. J Interv Cardiol 2019;2019:3579671.

24. Tang GHL, Zaid S, Gupta E, et al. Impact of initial Evolut transcatheter aortic valve replacement deployment orientation on final valve orientation and coronary reaccess. Circ Cardiovasc Interv 2019;12:e008044.

25. Yudi MB, Sharma SK, Tang GHL, et al. Coronary Angiography and Percutaneous Coronary Intervention After Transcatheter Aortic Valve Replacement. J Am Coll Cardiol 2018;71:1360-78.

26. Dvir D, Leipsic J, Blanke P, et al. Coronary obstruction in transcatheter aortic valve-in-valve implantation: preprocedural evaluation, device selection, protection, and treatment. Circ Cardiovasc Interv 2015;8:e002079.

27. Nour D, Allahwala U, Hansen P, et al. Cardiogenic shock due to late chimney stent failure following valve-in-valve transcatheter aortic valve replacement. J Am Coll Cardiol Case Rep 2019;1:313-8.

28. Khan JM, Dvir D, Greenbaum AB, et al. Trans- catheter laceration of aortic leaflets to prevent coronary obstruction during transcatheter aortic valve replacement. JACC Cardiovasc Interv 2018;11:677-89.

29. Fetahovic T, Hayman S, Cox S, et al. The prophylactic chimney snorkel technique for the prevention of acute coronary occlusion in high risk for coronary obstruction transcatheter aortic valve replacement/implantation cases. Heart Lung Circ 2019;28:e126-30.

30. Jabbour RJ, Imperial College London, United Kingdom, Tanaka A, et al. Delayed coronary occlusion after transcatheter aortic valve implantation: implications for new transcatheter heart valve design and patient management. Interv Cardiol 2018;13:137.

31. Palmerini T, Chakravarty T, Saia F, et al. Coronary Protection to Prevent Coronary Obstruction During Transcatheter Aortic Valve Replacement: A Multicenter International Registry. JACC Cardiovasc Interv 2020;13:739-47.

32. Jilaihawi $H$, Chen $M$, Webb J, et al. A bicuspid aortic valve imaging classification for the TAVR era. JACC Cardiovasc Imaging 2016;9:1145-58.
Cite this article as: Arshi A, Yakubov SJ, Stiver KL, Sanchez CE. Overcoming the transcatheter aortic valve replacement Achilles heel: coronary re-access. Ann Cardiothorac Surg 2020;9(6):468-477. doi: 10.21037/acs-2020-av-38 